\title{
Exact Solution of the Isovector Neutron-Proton Pairing Hamiltonian
}

\author{
J. Dukelsky, ${ }^{1}$ V. G. Gueorguiev, ${ }^{2,3}$ P. Van Isacker, ${ }^{4}$ S. Dimitrova, ${ }^{3}$ B. Errea, ${ }^{1}$ and S. Lerma H. ${ }^{1}$ \\ ${ }^{1}$ Instituto de Estructura de la Materia, CSIC. Serrano 123, 28006 Madrid, Spain \\ ${ }^{2}$ Lawrence Livermore National Laboratory, Livermore, California, USA \\ ${ }^{3}$ Institute of Nuclear Research and Nuclear Energy, BAS, Sofia 1784, Bulgaria \\ ${ }^{4}$ Grand Accélérateur National d'Ions Lourds, BP 55027, F-14076 Caen Cedex 5, France
}

(Received 28 November 2005; published 23 February 2006)

\begin{abstract}
The complete exact solution of the $T=1$ neutron-proton pairing Hamiltonian is presented in the context of the $\mathrm{SO}(5)$ Richardson-Gaudin model with nondegenerate single-particle levels and including isospin symmetry-breaking terms. The power of the method is illustrated with a numerical calculation for ${ }^{64} \mathrm{Ge}$ for a $p f+g_{9 / 2}$ model space which is out of reach of modern shell-model codes.
\end{abstract}

DOI: 10.1103/PhysRevLett.96.072503

Exactly solvable models (ESM) provide important insights into the structure of many-body quantum systems. The two main advantages of ESMs are: (1) they can describe in an analytical or exact numerical way a wide variety of elementary phenomena. (2) They can be and have been used as a testing ground for various many-body approaches.

A particular class of ESMs, extensively used in nuclear physics, are the dynamical-symmetry models. In this case the Hamiltonian can be expressed in terms of Casimir operators of a chain of nested algebras. An example often used to introduce nuclear superconductivity [see, e.g., Ref. [1]] is the rank-1 (Lie) algebra SU(2). Examples of dynamical-symmetry models associated with a rank-2 algebra are Elliott's SU(3) model of nuclear deformation [2] and the SO(5) model of $T=1$ isovector pairing between neutrons and protons [3] which has found many applications in nuclei [see, e.g., Ref. [4] ].

The concept of quantum integrability, closely linked with exact solvability, goes beyond the limits of the dynamical-symmetry approach. A quantum system is integrable if there exist as many commuting Hermitian operators (integrals of motion) as quantum degrees of freedom [5]. The set of Casimir operators of a chain of nested algebras satisfies this condition.

Dynamical-symmetry models are usually defined for degenerate single-particle levels. Lifting this degeneracy breaks the dynamical symmetry but may still preserve integrability. The pairing model with nondegenerate single-particle levels, of which an exact solution was found by Richardson in the 1960s [6], represents an example of an ESM with such characteristics. Recently, more general exactly solvable pairing models, both for fermions and for bosons, called Richardson-Gaudin (RG) models, have been proposed [7,8].

The RG pairing models are based on rank-1 algebras: $\mathrm{SU}(2)$ for fermions and $\mathrm{SU}(1,1)$ for bosons. In this Letter we carry out the first step in extending the RG models to higher-rank algebras by considering a RG model based on the rank-2 algebra $\mathrm{SO}(5)$. The model Hamiltonian de-
PACS numbers: 21.60.Fw, 03.65.-w, 27.50.+e, 74.20.Rp

scribes a two-component system consisting of neutrons and protons interacting through an isovector $(T=1)$ pairing force and distributed over nondegenerate orbits. This neutron-proton $(n p)$ pairing Hamiltonian with nondegenerate orbits has been studied by Richardson [9] who proposed an exact solution. However, it was shown subsequently that Richardson's solution is incorrect for more than two nucleon pairs [10] by explicitly solving the case of three-nucleon pairs. Independently, Links et al. derived an exact solution for the isospin invariant $\mathrm{SO}(5)$ model by making use of the quantum inverse scattering method [11].

We present here the most general exact solution of the RG SO(5) model including isospin symmetry-breaking terms and for states with arbitray seniority. In addition to the construction of the complete set of integrals of motion from which more general exactly solvable $n p$-pairing Hamiltonians can be derived, we present here the first numerical exact solution of the $\mathrm{SO}(5) \mathrm{RG}$ model for ${ }^{64} \mathrm{Ge}$ in a Hilbert space built from the $p f+g_{9 / 2}$ shells, of which the dimension goes well beyond the limits of modern shell-model codes based on exact diagonalization.

$\mathrm{SO}(5)$ has also been proposed as the symmetry underlying high- $T_{c}$ superconductivity [12]. The exactly solvable RG model discussed in this Letter may conceivably be used to generalize $\mathrm{SO}(5)$ condensed-matter models [13] by the explicit addition of nondegenerate single-particle symmetry-breaking terms. Other possible applications might be found in polarized ultracold Fermi gases with $p$-wave pairing interactions [14].

We begin by introducing the 10 generators of the $\mathrm{SO}(5)$ algebra in a representation well suited for nuclear physics problems. Let us define first the three $T=1$ pair-creation operators: $\hat{b}_{-1, i}^{\dagger}=\hat{n}_{i}^{\dagger} \hat{n}_{i}^{\dagger}, \hat{b}_{0, i}^{\dagger}=\left(\hat{n}_{i}^{\dagger} \hat{p}_{\bar{i}}^{\dagger}+\hat{p}_{i}^{\dagger} \hat{n}_{\bar{i}}^{\dagger}\right) / \sqrt{2}$, and $\hat{b}_{+1, i}^{\dagger}=\hat{p}_{i}^{\dagger} \hat{p}_{\bar{i}}^{\dagger}$, where $n$ and $p$ refer to neutrons and protons, respectively, and $i$ labels a single-particle basis (with $\bar{i}$ its time-reversed state) which may be associated with the spherical shell-model basis $i \equiv j m$ or with an axially symmetric deformed basis $i \equiv \alpha m$. The three pairannihilation operators are $\hat{b}_{-1, i}, \hat{b}_{0, i}$, and $\hat{b}_{+1, i}$. The three 
components of the isospin operator $\left[\hat{T}_{+, i}=\left(\hat{p}_{i}^{\dagger} \hat{n}_{i}+\right.\right.$ $\left.\hat{p}_{\bar{i}}^{\dagger} \hat{n}_{\bar{i}}\right) / \sqrt{2}, \quad \hat{T}_{0, i}=\left(\hat{p}_{i}^{\dagger} \hat{p}_{i}+\hat{p}_{\bar{i}}^{\dagger} \hat{p}_{\bar{i}}\right) / 2-\left(\hat{n}_{i}^{\dagger} \hat{n}_{i}+\hat{n}_{\bar{i}}^{\dagger} \hat{n}_{\bar{i}}\right) / 2$, and $\left.\hat{T}_{-, i}=\left(\hat{n}_{i}^{\dagger} \hat{p}_{i}+\hat{n}_{\bar{i}}^{\dagger} \hat{p}_{\bar{i}}\right) / \sqrt{2}\right]$ close the $\mathrm{SU}_{T}(2)$ subalgebra of $\mathrm{SO}(5)$. These 9 operators together with the number operator $\hat{N}_{i}=\hat{p}_{i}^{\dagger} \hat{p}_{i}+\hat{p}_{\bar{i}}^{\dagger} \hat{p}_{\bar{i}}+\hat{n}_{i}^{\dagger} \hat{n}_{i}+\hat{n}_{\bar{i}}^{\dagger} \hat{n}_{\bar{i}}$ define the $\mathrm{SO}(5)$ algebra.

For a system with $L$ single-particle states $i=1, \ldots, L$ there are $L$ integrals of motion:

$$
\begin{aligned}
\hat{R}_{i}= & (2+\Delta) \hat{H}_{i}+\Delta \hat{T}_{0, i}+2 g \sum_{i^{\prime}(\neq i)=1}^{L} \frac{1}{z_{i}-z_{i^{\prime}}} \\
& \times\left[\sum_{\mu}\left(\hat{b}_{\mu, i}^{\dagger} \hat{b}_{\mu, i^{\prime}}+\hat{b}_{\mu, i^{\prime}}^{\dagger} \hat{b}_{\mu, i}\right)+\hat{T}_{i} \cdot \hat{T}_{i^{\prime}}+\hat{H}_{i} \hat{H}_{i^{\prime}}\right],
\end{aligned}
$$

where $\hat{H}_{i}=\hat{N}_{i} / 2-1$. The expression (1) follows from the integrals of motion valid for any semisimple algebra of arbitrary rank [15]. Since $S O(5)$ is of rank 2, its Cartan subalgebra contains two elements in the chosen basis, namely $\hat{H}_{i}$ and $\hat{T}_{0, i}$, which appear linearly in (1). The set of $L$ parameters $z_{i}$ together with the two constants $g$ and $\Delta$ can be freely chosen and it is straightforward to check that the integrability condition $\left[\hat{R}_{i}, \hat{R}_{j}\right]=0$ is valid for any choice of the $L+2$ parameters. A simplified version of (1) was previously derived using the algebraic Bethe ansatz [11].

The eigenvalues of the integrals of motion are

$$
\begin{aligned}
r_{i}= & 2 g \sum_{i^{\prime}(\neq i)=1}^{L} \frac{\left(\frac{v_{i}}{2}-1\right)\left(\frac{v_{i^{\prime}}}{2}-1\right)+t_{i} t_{i^{\prime}}}{z_{i}-z_{i^{\prime}}}-g \sum_{\beta=1}^{M+T_{0}+t} \frac{2 t_{i}}{z_{i}-\omega_{\beta}} \\
& +g \sum_{\alpha=1}^{M} \frac{v_{i}+2 t_{i}-2}{z_{i}-e_{\alpha}}+\left[(2+\Delta)\left(\frac{v_{i}}{2}-1\right)-\Delta t_{i}\right],
\end{aligned}
$$

where $e_{\alpha}$ and $\omega_{\beta}$ are solutions of the equations

$$
\begin{aligned}
& \frac{1}{g}=\frac{1}{2} \sum_{i=1}^{L} \frac{v_{i}+2 t_{i}-2}{z_{i}-e_{\alpha}}+\sum_{\alpha^{\prime}(\neq \alpha)=1}^{M} \frac{2}{e_{\alpha^{\prime}}-e_{\alpha}}-\sum_{\beta=1}^{M+T_{0}+t} \frac{1}{\omega_{\beta}-e_{\alpha}}, \\
& \frac{\Delta}{g}=\sum_{\beta^{\prime}(\neq \beta)=1}^{M+T_{0}+t} \frac{2}{\omega_{\beta^{\prime}}-\omega_{\beta}}-\sum_{\alpha=1}^{M} \frac{2}{e_{\alpha}-\omega_{\beta}}-\sum_{i=1}^{L} \frac{2 t_{i}}{z_{i}-\omega_{\beta}} .
\end{aligned}
$$

The meaning of the quantum numbers appearing in (2) and (3) is as follows: $v_{i}$ is the seniority of each $i$ level, i.e., the number of fermions not paired in time-reversed states with isospin $T=1, t_{i}$ is the isospin of the unpaired fermions [this quantum number is often called reduced isospin [16]], $t=\sum_{i} t_{i}, M$ is the number of $T=1$ time-reversed pairs, and $T_{0}$ is the $z$ component of the total isospin, i.e., the eigenvalue of the operator $\hat{T}_{0}=\sum_{i} \hat{T}_{0, i}$. The total number of nucleons is $N=N_{p}+N_{n}=2 M+\sum_{i} v_{i}$, whereas their difference is $N_{p}-N_{n}=2 T_{0}$. The quantum numbers $M$, $T_{0}, v_{i}$, and $t_{i}$ are conserved; $T$ is also conserved if $\Delta=0$.

Although any function of the $\hat{R}_{i}$ can be used as an integrable Hamiltonian, the linear combination $\sum_{i=1}^{L} z_{i} \hat{R}_{i}$ yields simple expressions for the $n p$-pairing Hamiltonian and its corresponding eigenvalues:

$$
\begin{aligned}
\hat{H} & =\frac{1}{2} \sum_{i=1}^{L} z_{i} \hat{R}_{i}+g \hat{C} \\
& =\sum_{j} \varepsilon_{j}\left(\hat{N}_{j}+\Delta \hat{N}_{p, j}\right)+\frac{g}{2} \hat{T} \cdot \hat{T}+g \sum_{\mu j m j^{\prime} m^{\prime}} \hat{b}_{\mu, j m}^{\dagger} \hat{b}_{\mu, j^{\prime} m^{\prime}},
\end{aligned}
$$

where $\hat{C}$ is a constant operator depending on the conserved quantities. We have introduced the variables $\varepsilon_{j}=z_{j} / 2$ and specialized to a spherical basis $i \equiv j m$. The second term on the right-hand side of (1) breaks the isospin symmetry. For $\Delta \neq 0$ the operator $\hat{T}^{2}$ does not commute with the Hamiltonian (4) and, consequently, $T$ is not a good quantum number but $T_{0}$ is still a conserved quantity. The same linear combination of the $r_{i}$ and the use of the Richardson equations (3) yield the eigenvalues of (4):

$$
\begin{aligned}
E= & \sum_{\alpha=1}^{M} e_{\alpha}+\frac{\Delta}{2} \sum_{\beta=1}^{M+T_{0}+t} \omega_{\beta}+\sum_{j} \varepsilon_{j}\left[\frac{v_{j}}{2}(2+\Delta)-\Delta t_{j}\right] \\
& +\frac{g}{2} T_{0}\left(T_{0}-1\right) .
\end{aligned}
$$

Each solution of the Eq. (3) gives an eigenstate of the $n p$-pairing Hamiltonian. The spectral parameters $e_{\alpha}$ are interpreted as pair energies as in the case of $\mathrm{SU}(2)$ pairing. However, due to the larger rank of $\mathrm{SO}(5)$, a new set of spectral parameters $\omega_{\beta}$ appears in the Eq. (3). These new parameters are associated with the $\mathrm{SU}_{T}(2)$ isospin subalgebra and there are $M+T_{0}+t$ of them. In the limit $\Delta=0$ the number of finite $\omega_{\beta}$ parameters reduces to $M+t-T$ for each possible isospin $T$. The Bethe ansatz for the $\mathrm{SO}(5)$ eigenstates of the RG model is a product wave function [17]:

$$
\left[\prod_{\alpha=1}^{M} \hat{b}_{-1}^{\dagger}\left(e_{\alpha}\right)\right]^{M+T_{0}+t}\left[\hat{T}_{+=1}\left(\omega_{\beta}\right)-\sum_{\alpha=1}^{M} \frac{\hat{I}_{+, \alpha}}{\omega_{\beta}-e_{\alpha}}\right]|\Lambda\rangle,
$$

where the spectral dependence of the operators is

$$
\hat{T}_{+}\left(\omega_{\beta}\right)=\sum_{i} \frac{\hat{T}_{+, i}}{2 \varepsilon_{i}-\omega_{\beta}}, \quad \hat{b}_{\mu}^{\dagger}\left(e_{\alpha}\right)=\sum_{i} \frac{\hat{b}_{\mu, i}^{\dagger}}{2 \varepsilon_{i}-e_{\alpha}},
$$

and $\hat{I}_{+, \alpha}$ is a raising operator for $\hat{b}_{\mu}^{\dagger}\left(e_{\alpha}\right): \hat{b}_{\mu}^{\dagger}\left(e_{\alpha}\right) \hat{I}_{+, \alpha}=$ $\hat{b}_{\mu+1}^{\dagger}\left(e_{\alpha}\right)$ for $\mu=-1,0, \hat{b}_{+1}^{\dagger}\left(e_{\alpha}\right) \hat{I}_{+, \alpha}=0$, and $|\Lambda\rangle$ is a lowest-weight state defined by $\hat{b}_{\mu, i}|\Lambda\rangle=\hat{T}_{-, i}|\Lambda\rangle=0$. To show the behavior of the spectral parameters $e_{\alpha}$ and $\omega_{\beta}$ as a function of the isospin-breaking term $\Delta$, we plot in Fig. 1 some selected solutions of the Richardson equations (3) for a system of two neutrons and two protons in two shells $\left(j_{0}=1 / 2\right.$ and $\left.j_{1}=3 / 2\right)$ within the seniority-0 subspace $\left(M=2, T_{0}=0, t_{i}=v_{i}=0\right)$.

For $\Delta=0$ there are two finite $\omega_{\beta}$ complex conjugate parameters for $T=0$ while the two pair energies are real 
and negative, for $T=1$ there is one real, finite $\omega_{\beta}$ and two real pair energies $e_{\alpha}$, and, finally, the $T=2$ case reduces to $\mathrm{SU}(2)$ for like particles with two $e_{\alpha}$ parameters forming a complex conjugate pair. For finite $\Delta$ there is isospin mixing and the number of finite parameters $\omega_{\beta}$ is always two. Figure 1 thus confirms that the number of finite $\omega_{\beta}$ spectral parameters reduces from $M=2$ to $M-T$ when $\Delta \rightarrow 0$. For $T=1$ one of the real $\omega_{\beta}$ goes to $-\infty$ in this limit, vanishing from the Richardson equations (3) but giving a finite contribution to the Hamiltonian eigenvalues (4) [18]. Analogously, in the $T=2$ case the two $\omega$ parameters tend to $\infty$ in the $\Delta=0$ limit. Also shown are the energies of the three eigenstates of the $n p$-pairing Hamiltonian (4). We emphasize that, while these are the eigenvalues of a particular Hamiltonian, the spectral parameters completely define the eigenfunction (6) of the $L$ integrals of motion (1) from which $\hat{H}$ is constructed and their corresponding eigenvalues (2).

We now turn to the discussion of a numerical calculation for ${ }^{64} \mathrm{Ge}$. We consider a model space that is well beyond modern shell-model capabilities based on exact diagonalization: 12 valence neutrons and 12 valence protons with a ${ }^{40} \mathrm{Ca}$ core. The adopted single-particle energies are (in $\mathrm{MeV}) \varepsilon_{f_{7 / 2}}=0.00, \varepsilon_{p_{3 / 2}}=6.00, \varepsilon_{f_{5 / 2}}=6.25, \varepsilon_{p_{1 / 2}}=$ 7.1 , and $\varepsilon_{g_{9 / 2}}=9.60$, and two pairing strengths, $g=$
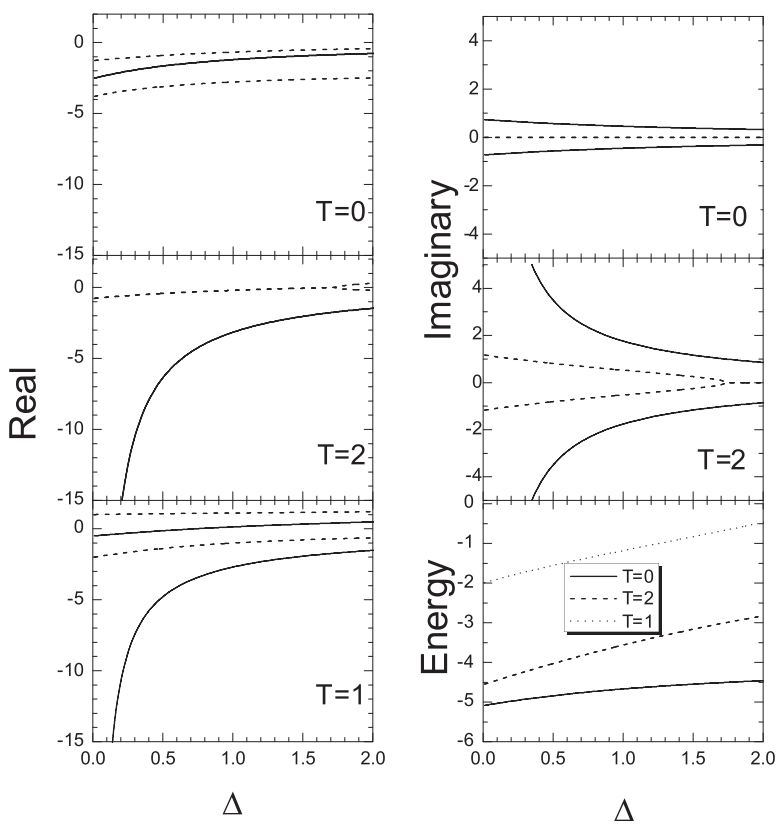

FIG. 1. Pair energies $e_{\alpha}$ (dashed lines) and spectral parameters $\omega_{\beta}$ (solid lines) as a function of the parameter $\Delta$ for three different states of a $2 n-2 p$ system with two shells $\left(j_{0}=1 / 2\right.$ and $j_{1}=3 / 2$ ) in the seniority-0 subspace. The label $T$ refers to the isospin in the limit $\Delta=0$. The figure shows the lowestenergy states of the Hamiltonian (4) for each $T$ with energies plotted in the bottom-right panel. Since the spectral parameters of the $T=1$ state are real, their imaginary part is not shown. The interaction strength and single-particle energies are $g=-1$, $\varepsilon_{0}=0$, and $\varepsilon_{1}=1$.
-0.05 (weak) and -0.5 (strong), are considered. We assume isospin symmetry $(\Delta=0)$ and consider the seniority-0 subspace.

Results for the lowest $T=0,1$, and 2 states are shown in Fig. 2. The $T=0$ solution corresponds to the ground state while the $T=1$ and $T=2$ solutions are excited states in ${ }^{64} \mathrm{Ge}$. As in SU(2), the different configurations can be classified in the weak-coupling limit. At weak coupling ( $g \rightarrow 0$ ) eight pairs occupy the $f_{7 / 2}$ level and four pairs are in the $p_{3 / 2}$ level for the state with $T=0$. This is reflected in the upper left panel of Fig. 2 where 8 pair energies appear close to $2 \varepsilon_{f_{7 / 2}}$ and 4 pair energies are close to $2 \varepsilon_{p_{3 / 2}}$ making the corresponding terms in (7) dominant. Because of the Pauli principle, this configuration is not allowed for a state with $T=1$ and, correspondingly, one pair energy is close to $2 \varepsilon_{f_{5 / 2}}$. In all cases the $\omega_{\beta}$ parameters are intertwined with the pair energies $e_{\alpha}$. The number of $\omega_{\beta}$ parameters $(M-T)$, together with the initial configuration at weak coupling, defines each eigenstate of the $n p$-pairing Hamiltonian. As $|g|$ increases, the $e_{\alpha}$ and $\omega_{\beta}$ parameters expand in the complex plain. The solutions are subject to numerical instabilities due to singularities arising when a real pair energy $e_{\alpha}$ crosses a single-particle energy or when real $e_{\alpha}$ and $\omega_{\beta}$ parameters cross. An
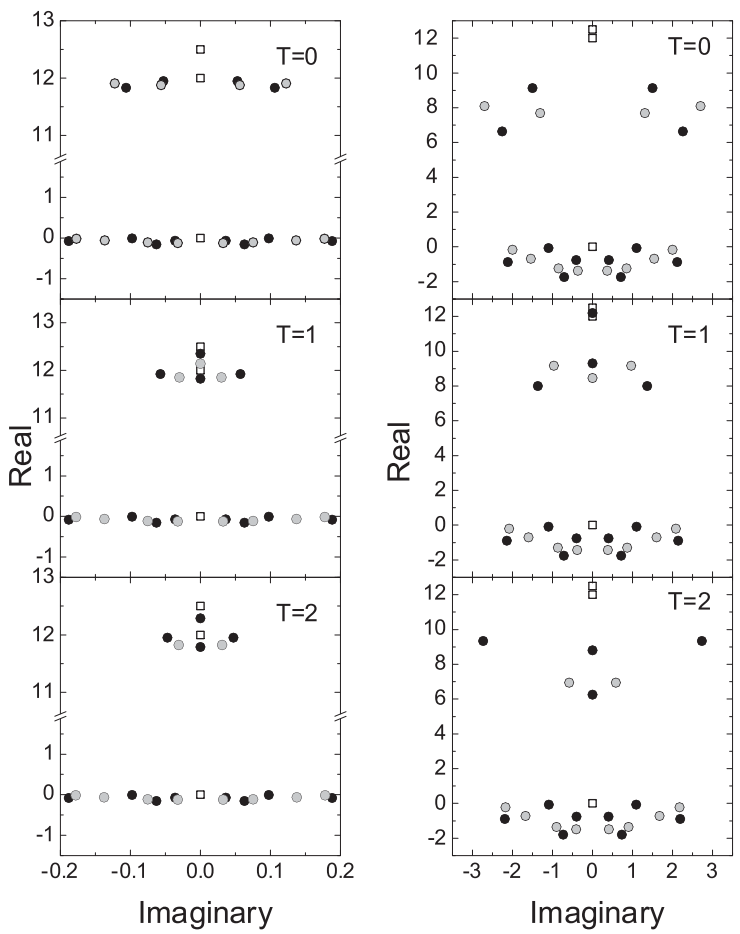

FIG. 2. Complex-plane representation of the pair energies $e_{\alpha}$ and spectral parameters $\omega_{\beta}$ for the lowest-energy states with isospin $T=0,1,2$ in ${ }^{64} \mathrm{Ge}$. The left panel corresponds to weak coupling $g=-0.05$ and the right panel to strong coupling $g=$ -0.5 . The squares represent the three lowest single-particle energies $\left(2 \varepsilon_{f_{7 / 2}}=0.00,2 \varepsilon_{p_{3 / 2}}=12,2 \varepsilon_{f_{5 / 2}}=12.5\right)$, the black circles are the pair energies $e_{\alpha}$, and the gray circles are the parameters $\omega_{\beta}$. All energies are in $\mathrm{MeV}$. 

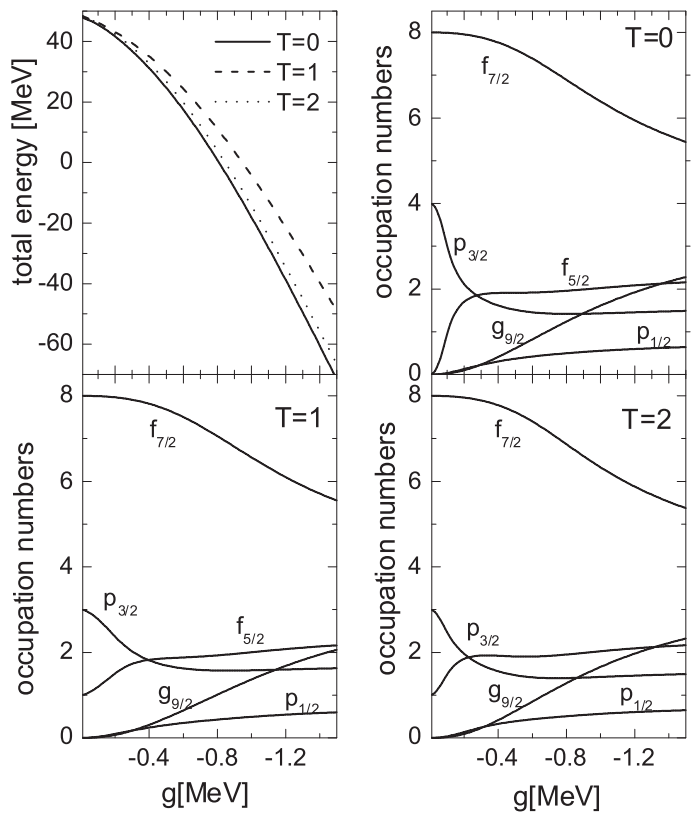

FIG. 3. Eigenenergies and occupation probabilities of singleparticle levels of the $T=0,1$, and 2 states in ${ }^{64} \mathrm{Ge}$ as a function of the pairing strength $g$.

example of the first class of crossings can be observed in Fig. 2 for $T=2$ where the pair energy above the $p_{3 / 2}$ level at weak coupling goes down with increasing $g$ and crosses the $p_{3 / 2}$ single-particle energy. The $T=1$ case shows an exchange of positions on the real axis of a pair energy $e_{\alpha}$ and a $\omega_{\beta}$ parameter as an example of the second class of singularities. The first class of singularities was already present in SU(2) pairing and precluded the practical use of Richardson's solution for a long time. Recently, a new method to overcome this numerical problem was proposed [19]. We believe that the same procedure can be used to treat the second class of singularities as well, allowing the exact solution of the $\mathrm{SO}(5)$ model for very large systems.

As a further illustration of the method we show in Fig. 3 the eigenenergies and the occupation probabilities of single-particle levels as a function of the pairing strength. The occupation probabilities can be obtained making use of Hellmann-Feynman theorem which expresses them in terms of derivatives of the eigenvalues of the integrals of motion $r_{i}$ as $\left\langle N_{p, i}\right\rangle=1+\frac{\partial r_{i}}{\partial \Delta}$, and $\left\langle N_{n, i}\right\rangle=r_{i}-g \frac{\partial r_{i}}{\partial g}-$ $(1+\Delta)\left(1+\frac{\partial r_{i}}{\partial \Delta}\right)+1$. These derivatives can be related to the derivatives of the spectral parameters $e_{\alpha}$ and $\omega_{\alpha}$, which in turn can be obtained taking the derivatives of the Richardson equations (3).

In summary, as an application of generalized RG models, we have presented the complete solution of the $\mathrm{SO}(5)$ isovector $n p$-pairing problem. The generalization allows the introduction of one-body symmetry-breaking terms, such as nondegenerate single-particle energies, yielding an exact solution of the $\mathrm{SO}(5) n p$-pairing model for arbi- trary seniorities even if it includes an isospin-breaking term. The numerical solution of the $\mathrm{SO}(5)$ Richardson equations was presented for the specific example of ${ }^{64} \mathrm{Ge}$, together with a discussion of the behavior of the spectral parameters for weak and strong pairing. With this work the exact solution for large systems with $\mathrm{SO}(5)$ symmetry is now available which could be of great importance in condensed-matter physics when addressing the phenomenon of high- $T_{c}$ superconductivity [12,13]. Finally, the treatment of higher-rank algebras like $\mathrm{Sp}(6)$ and $\mathrm{SO}(8)$ opens the possibility of exact nuclear structure calculations with more realistic quantum integrable models.

This work was supported in part by the Spanish DGI under Grant No. BFM2003-05316-C02-02, by Bulgarian Contracts $\Phi-1416$ and $\Phi-1501$, and by a CICYTIN2P3 cooperation. V. G. G. acknowledges financial support from NATO and from the US DOE under Grant No. W-7405-Eng-48. S. L. H. was supported by the Spanish SEUI-MEC and B.E. was supported by the Spanish CE-CAM.

[1] P. Ring and P. Schuck, The Nuclear Many Body Problem (Springer-Verlag, Berlin, 1980).

[2] J. P. Elliott, Proc. Roy. Soc. London A 245, 128 (1958); 245, 562 (1958).

[3] B. H. Flowers, Proc. Roy. Soc. London A 212, 248 (1952).

[4] J. Engel, K. Langanke, and P. Vogel, Phys. Lett. B 389, 211 (1996).

[5] W. M. Zhang and D. H. Feng, Phys. Rep. 252, 1 (1995).

[6] R. W. Richardson, Phys. Lett. 3, 277 (1963); Phys. Rev. 141, 949 (1966).

[7] J. Dukelsky, C. Esebbag, and P. Schuck, Phys. Rev. Lett. 87, 066403 (2001).

[8] J. Dukelsky, S. Pittel, and G. Sierra, Rev. Mod. Phys. 76, 643 (2004).

[9] R. W. Richardson, Phys. Rev. 144, 874 (1966).

[10] F. Pan and J. P. Draayer, Phys. Rev. C 66, 044314 (2002).

[11] J. Links, H.-Q. Zhou, M. D. Gould, and R. H. McKenzie, J. Phys. A 35, 6459 (2002).

[12] E. Demler, W. Hanke, and S.-C. Zhang, Rev. Mod. Phys. 76, 909 (2004).

[13] L.-A. Wu, M. Guidry, Y. Sun, and C.-L. Wu, Phys. Rev. B 67, 014515 (2003).

[14] V. Gurarie, L. Radzihovsky, and A. V. Andreev, Phys. Rev. Lett. 94, 230403 (2005).

[15] M. Asorey, F. Falceto, and G. Sierra, Nucl. Phys. B622, 593 (2002).

[16] K. T. Hecht, Phys. Rev. 139, B794 (1965).

[17] A.G. Ushveridize, Quasi-Exactly Solvable Models in Quantum Mechanics (Institute of Physics, Bristol, 1994).

[18] It can be shown that in the limit $\Delta \rightarrow 0$ the second term for the diverging $\omega^{\prime} s$ and the fourth term in (5) combine to give $\frac{g}{2} T(T+1)$.

[19] S. Rombouts, D. Van Neck, and J. Dukelsky, Phys. Rev. C 69, 061303 (2004). 